\title{
BMJ Open Effect of repetitive transcranial magnetic stimulation on patients with severe depression: a study protocol for systematic review and meta-analysis of randomised clinical trials
}

\author{
Fang Han (D) , ${ }^{1}$ Shuai Tao, ${ }^{2}$ Shanshan Liang, ${ }^{3}$ Danyang Li, ${ }^{1}$ Yutong Me, ${ }^{4}$ \\ Hongyu Fan, ${ }^{1}$ Hao Wu, ${ }^{1}$ Gaofeng Zhang ${ }^{5}$
}

To cite: Han F, Tao S, Liang S, et al. Effect of repetitive transcranial magnetic stimulation on patients with severe depression: a study protocol for systematic review and meta-analysis of randomised clinical trials. BMJ Open 2021;11:e050098. doi:10.1136/ bmjopen-2021-050098

- Prepublication history and additional supplemental material for this paper are available online. To view these files, please visit the journal online (http://dx.doi.org/10.1136/ bmjopen-2021-050098).

FH and ST contributed equally.

Received 09 February 2021 Accepted 17 November 2021

Check for updates

(C) Author(s) (or their employer(s)) 2021. Re-use permitted under CC BY-NC. No commercial re-use. See rights and permissions. Published by BMJ.

For numbered affiliations see end of article.

Correspondence to Dr Fang Han; hanfang20201124@163.com and

Dr Gaofeng Zhang;

Zhanggaofeng7677@163.com

\section{ABSTRACT}

Introduction Depression is characterised by easy recurrence, high disability and high burden, and antidepressant therapy is the standard treatment. However, its treatment effect on patients with severe depressive disorder has been unsatisfactory. Previous studies have shown that repetitive transcranial magnetic stimulation (rTMS), as a neurotherapy, can effectively mitigate the severity of depressive symptoms. Yet, more evidence is still required for TMS to treat severe depression. This study will be the first systematic review of the efficacy and tolerability of TMS for treating severe depression. We expect it to guide future clinical practice of TMS for the treatment of psychiatric disorders.

Methods and analysis We will search for the randomised controlled trial (RCT) involving rTMS for treating depression in eight electronic databases, including PubMed, Web of Science, EMBASE, the Cochrane Library and Wanfang Database, from publication up to September 2021. We will define Improvement in depressive symptoms, the difference between pretreatment (baseline) and posttreatment as the primary outcomes. The difference between pretreatment and post-treatment changes in resting state $\mathrm{fMRI}$ will be regarded as the secondary outcomes. Quality assessment of the included articles will be independently performed according to the Cochrane Risk of Bias tool.

Ethics and dissemination Ethical approval is not essential because there is no need to collect individual patient data. And this study will be published in a peerreviewed journal.

Trial registration number CRD42020211460.

\section{INTRODUCTION}

Depression, featuring easy relapse, high disability rate and heavy burden, is a common mental system disease with a long course. Depression affects more than 300 million people in the world. Based on the WHO, depression is an immense burden of non-fatal health consequences, accounting for approximately $12 \%$ of the total life of disability. ${ }^{1-3}$
Strengths and limitations of this study

- This study will provide the first systematic review and meta-analysis for evaluating the efficacy of repetitive transcranial magnetic stimulation (rTMS) for severe depression.

- Since most randomised controlled trials (RCTs) on rTMS for severe depression have a small sample size, the present study will provide more reliable evidence for clinical management.

- The electronic search will only include RCTs published in Chinese and English that could limit the inclusion of studies.

It usually requires long-term maintenance therapy to prevent future emotional episodes as the primary goal of treatment. Antidepressant therapy is a standard method for the treatment of depression. ${ }^{4}$ However, it does not work for everyone with depression. Depression in these patients is usually termed as severe depressive disorder (SDD), which is defined as after two or more antidepressant treatments, the 17-item Hamilton Depression Rating Scale (HAMD-17) score reduction rate was less than $20 \% .^{56}$

The traditional treatment for SDD is facing challenges. Patients' quality of life can be significantly compromised, including sleep and work. Although pharmacotherapy and psychotherapy are effective for depression nowadays, only $33 \%$ of patients achieve a complete remission with medication during treatment in the acute phase, with less than $50 \%$ of patients failing to achieve remission after multiple medication trials. ${ }^{78}$ Besides, these treatments often do not work with the depressive symptoms. However, approximately $30 \%$ of patients with depression receive extensive psychotherapy (although 
around $20 \%$ in 2006 received psychotherapy). Previous resistance to antidepressants also reduces the likelihood of responding to subsequent interventions. Thus, many patients require better alternative treatment options for depression.

Repetitive transcranial magnetic stimulation (rTMS) was first demonstrated to treat depression in the mid1990s as a neurotherapy. rTMS uses the principle of induction to transmit electric current to the brain through the resistance layer of the scalp, skull and meninges, where it can change the electrical environment of neurons and cause neurons to discharge. ${ }^{9}{ }^{10}$ rTMS is applied to the prefrontal cortex and induces magnetic fields, leading to depolarisation of potential neurons and regulation of neural circuits involved in emotion regulation and depressive symptoms. ${ }^{11-13}$ Due to its advantages, including being non-invasive, safe, with minimal discomfort and convenient operation, rTMS has captured the attention of scientists, clinicians, and lay observers since its commercial advent in $1985 .{ }^{10}$ In addition, a metasystematic review including 18 studies has shown that accelerated TMS is effective in improving depressive symptom severity. ${ }^{14}$

However, doubts about rTMS's effectiveness in treating severe depression still need to be cleared with more evidence. Since the relevant evidence is rare, in our study, we will perform the first systematic review and meta-analysis to investigate the efficacy and tolerance of rTMS in the treatment of severe depression. Based on our summary of the literature, we expect it can guide the clinical practice of rTMS on the treatment of mental system disease in the future.

\section{METHODS}

\section{Patient and public involvement}

Patients or the public were not involved in the design, conduction, reporting or dissemination plans of our research.

\section{Study registration}

The protocol of this review will be conducted and reported referring to the Preferred Reporting Items for Systematic Reviews and Meta-Analysis Protocols (PRISMA-P) statement guidelines (see online supplemental appendix 1). The protocol has been registered on the PROSPERO.

\section{Study selection}

Type of studies

We will evaluate the articles based on criteria of the review target and participants, interventions, comparisons and outcomes. Only randomised and controlled trials involving participants with TMS versus other treatments, placebo or sham treatment were included in this review. The language of the literature will be limited to Chinese and English. Studies that mentioned the term 'randomisation' will be considered. Other designs such as case reports and non-randomised controlled trials will be excluded. Studies that used incorrect randomisation methods (eg, pseudorandomisation) will be excluded.

Type of participants

Inclusion criteria

(1) Subjects referring to the diagnostic and Statistical Manual of mental disorders (4th edition), the diagnostic criteria of the depressive episode were met; (2) subjects were assessed with Hamilton Depression Scale Item 3, and score $\geq 2$ points ${ }^{15}$; (3) subjects were assessed with Suicidal Ideation Scale, and score $>12$ points, but refused to accept point convulsion treatment ${ }^{16}$; (4) subjects signed informed consent.

\section{Exclusion criteria}

(1) Participants with a history of severe physical disease, organic brain disease, depression caused by other psychoactive substances and independent substances (eg, alcohol or drugs); (2) participants with brain surgery and epilepsy history; (3) participants who adjust drug dosage during treatment; (4) participants with metal or electronic devices built into the body; (5) participants who have been treated with modified electroconvulsive therapy in the past 3 months; (6) participants in pregnancy or breastfeeding period, and those who refused to sign the informed consent.

\section{Type of interventions}

We will consider studies evaluating the treatment of rTMS (with different frequencies in $\mathrm{Hz}$, stimulation intensity, total stimuli, pulses per session, sessions per day, intersession interval in minutes, trains per session, the intertrain interval in seconds).

\section{Type of comparators}

We will include and categorise the comparators in the study as follows: (1) rTMS versus sham rTMS, (2) rTMS versus placebo, (3) rTMS versus waiting list/usual care/ no treatment. Articles comparing different rTMS will be removed.

\section{Outcome measures}

\section{Primary outcomes}

We will define improvement in depressive symptoms (eg, decline on the HAMD or Suicidal Ideation Scale Score), that is, the difference between pretreatment (baseline) and post-treatment, as the primary outcomes.

\section{Secondary outcomes}

Except for the changes in resting state fMRI, other differences before and after treatment will be considered as secondary results.

\section{Search strategies}

An electronic search will be carried out in the following databases: PubMed, Web of Science, EMBASE, the Cochrane Library and WAN FANG from publication up to September 2021. We will use the terms of medical subjects $(\mathrm{MeSH})$ and keywords individually or in combination on 
PubMed (see online supplemental appendix 2) during the query. However, the search strategy for other databases will be slightly modified.

\section{Study selection}

Two reviewers will assess the titles and abstracts of all studies separately for use by potential candidates. Reduplicated studies will be deleted. After screening the titles and abstracts, full-text copies of all eligible studies will be downloaded for reassessment. In the event that a reviewer is uncertain about the eligibility of any study, the full text will be acquired for re-examination. In case of disagreement, a third reviewer will be consulted. Excluded studies and reasons for exclusion will be recorded. The specific research screening process will be shown in the PRISMA flow diagram.

\section{Data extraction}

Two investigators will perform data extraction independently. Characteristics of studies, participants, methods, interventions, results, outcomes, adverse events, conflicts of interest, ethical recognition and other necessary information will be extracted. If the reported data are insufficient, the corresponding authors or relevant authors will be contacted by email. Besides, any disagreement will be settled by discussion between the two authors, and a third author will be invited for further judgement of the disputes.

\section{Risk of bias assessment}

The authors will assess the risk of bias using RoB V.2.0 of Cochrane Collaboration Evaluation of all included studies. ${ }^{17}$ The following areas of bias risk will be assessed: sequence generation, hidden distribution of sequences, blinding of participants, personnel and outcome evaluators, incomplete result data, selective results reporting and other sources of bias. The judgement of these projects will be divided into three levels: 'low risk of bias', 'high risk of bias' and 'unclear risk of bias'. Conflicts of any differences will be discussed, or another reviewer will review these conflicts to facilitate a consensus.

\section{Quality of evidence assessment}

According to the recommended evaluation, development and evaluation classification method, evaluating the quality of evidence for important outcomes can be divided into four levels: high quality, medium quality, low quality and very low quality. ${ }^{18} 19$ The basis of risk of bias, inconsistency, indirectness, inaccuracy and publication bias is the way how evidence quality is generally judged.

\section{Measures of effects}

We will use the Review Manager software V.5.3 to carry out the statistical analysis. Mean difference (MD) or standardised MD will be used for continuous data. Risk ratio (RR) or risk difference will be used to analyse dichotomous data. Each parameter's corresponding 95\% CI will be calculated between the treatment and control groups.

\section{Assessment of heterogeneity}

We leveraged Cochrane's $Q$ test to examine whether significant interstudy heterogeneity exists. It is assessed via the computed $\mathrm{I}^{2}$ statistic, where values $>50 \%$ indicated moderate heterogeneity. We employed a random effects model to calculate summary $\mathrm{MD}$ and $\mathrm{RR}$ and $95 \%$ CI regardless of heterogeneity.

\section{Assessment of reporting bias}

The reporting bias will be evaluated using a funnel plot when more than 10 tests are included.

\section{Sensitivity analysis}

Monitoring the robustness of the primary decision made in the review process can be the sensitivity analysis results. Multiple decision points will be taken, such as sample size, methodological weakness and missing data. Finally, we will discuss the risk of bias in the review process indicated by the sensitivity analysis results.

\section{Subgroup analysis}

When data are available and considerable heterogeneity is detected, a subgroup analysis will be carried out consulting variations in the characteristics of the trial participants, rTMS treatment, the amplitude of low-frequency fluctuation and functional connectivity density.

\section{Ethics and dissemination}

No ethical approval is required because the publications included in our study do not involve patients' privacy. The primary data will be from the published literature. This study does not involve human participants.

\section{Author affiliations}

${ }^{1}$ Department of Radiology, Affiliated Zhongshan Hospital of Dalian University, Dalian, Liaoning, China

${ }^{2}$ Dalian Key Laboratory of Smart Medical and Health, Dalian University, Dalian, China ${ }^{3}$ Affiliated Zhongshan Hospital of Dalian University, Dalian, China

${ }^{4}$ Department of Clinical Medicine, Dalian University, Dalian, China

${ }^{5}$ Affiliated Hospital of Zunyi Medical University, Zunyi, Guizhou, China

Contributors FH and HW conceived the idea for this study. ST provided statistical advice and input. SL and DL drafted the study. YM, HF and GZ reviewed the study and provided critical feedback.

Funding This work was supported by Post-doctoral Scientific Research Fund (303044) and National Key R\&D Program of China (2018YFC2001700).

Competing interests None declared.

Patient consent for publication Not applicable.

Provenance and peer review Not commissioned; externally peer reviewed.

Supplemental material This content has been supplied by the author(s). It has not been vetted by BMJ Publishing Group Limited (BMJ) and may not have been peer-reviewed. Any opinions or recommendations discussed are solely those of the author(s) and are not endorsed by BMJ. BMJ disclaims all liability and responsibility arising from any reliance placed on the content. Where the content includes any translated material, BMJ does not warrant the accuracy and reliability of the translations (including but not limited to local regulations, clinical guidelines, terminology, drug names and drug dosages), and is not responsible for any error and/or omissions arising from translation and adaptation or otherwise.

Open access This is an open access article distributed in accordance with the Creative Commons Attribution Non Commercial (CC BY-NC 4.0) license, which permits others to distribute, remix, adapt, build upon this work non-commercially, and license their derivative works on different terms, provided the original work is 
properly cited, appropriate credit is given, any changes made indicated, and the use is non-commercial. See: http://creativecommons.org/licenses/by-nc/4.0/.

\section{ORCID iD}

Fang Han http://orcid.org/0000-0002-8520-3776

\section{REFERENCES}

1 Wang PS, Aguilar-Gaxiola S, Alonso J, et al. Use of mental health services for anxiety, mood, and substance disorders in 17 countries in the who world mental health surveys. Lancet 2007;370:841-50.

2 Arensman E, Scott V, De Leo D, et al. Suicide and suicide prevention from a global perspective. Crisis 2020;41:S3-7.

3 Gonda X, Petschner P, Eszlari N, et al. Genetic variants in major depressive disorder: from pathophysiology to therapy. Pharmacol Ther 2019;194:22-43.

4 Kok RM, Reynolds CF. Management of depression in older adults: a review. JAMA 2017;317:2114-22.

5 Husain MI, Chaudhry IB, Khoso AB, et al. Minocycline and celecoxib as adjunctive treatments for bipolar depression: a multicentre, factorial design randomised controlled trial. Lancet Psychiatry 2020;7:515-27.

6 Mclntyre R, Kennedy S, Bagby RM, et al. Assessing full remission. $J$ Psychiatry Neurosci 2002;27:235-9.

7 Rush AJ, Trivedi MH, Wisniewski SR, et al. Acute and longer-term outcomes in depressed outpatients requiring one or several treatment steps: a STAR*D report. Am J Psychiatry 2006;163:1905-17.

8 Nelson JC. The STAR*D study: a four-course meal that leaves us wanting more. Am J Psychiatry 2006;163:1864-6.

9 Pascual-Leone A, Rubio B, Pallardó F, et al. Rapid-Rate transcranial magnetic stimulation of left dorsolateral prefrontal cortex in drugresistant depression. Lancet 1996;348:233-7.
10 Wassermann EM, Zimmermann T. Transcranial magnetic brain stimulation: therapeutic promises and scientific gaps. Pharmacol Ther 2012;133:98-107.

11 Salomons TV, Dunlop K, Kennedy SH, et al. Resting-State cortico-thalamic-striatal connectivity predicts response to dorsomedial prefrontal rTMS in major depressive disorder. Neuropsychopharmacology 2014;39:488-98.

12 Liston C, Chen AC, Zebley BD, et al. Default mode network mechanisms of transcranial magnetic stimulation in depression. Biol Psychiatry 2014;76:517-26.

13 George MS, Wassermann EM, Williams WA, et al. Daily repetitive transcranial magnetic stimulation (rTMS) improves mood in depression. Neuroreport 1995;6:1853-6.

14 Sonmez Al, Camsari DD, Nandakumar AL, et al. Accelerated TMS for depression: a systematic review and meta-analysis. Psychiatry Res 2019;273:770-81.

15 Zimmerman M, Martinez JH, Young D, et al. Severity classification on the Hamilton depression rating scale. J Affect Disord 2013;150:384-8.

16 McCall WV, Benca RM, Rosenquist PB, et al. Reducing suicidal ideation through insomnia treatment (REST-IT): a randomized clinical trial. Am J Psychiatry 2019;176:957-65.

17 Higgins JPT, Altman DG, Gøtzsche PC, et al. The Cochrane collaboration's tool for assessing risk of bias in randomised trials. BMJ 2011;343:d5928.

18 Puhan MA, Schünemann HJ, Murad MH, et al. A grade Working group approach for rating the quality of treatment effect estimates from network meta-analysis. BMJ 2014;349:g5630.

19 Guyatt G, Oxman AD, Akl EA, et al. Grade guidelines: 1. IntroductionGRADE evidence profiles and summary of findings tables. J Clin Epidemiol 2011;64:383-94. 